\title{
SigNificAdOS DEL CUERPO Y LA SALUd EN EL CONTEXTO DE LOS ESTILOS DE VIDA MODERNOS
}

\author{
Dania Oralia Aguilar Martínez \\ Universidad Autónoma de la Ciudad de México \\ Recibido: 10 de enero del 2020 / Aceptado: 2 de abril del 2020 \\ doi: 10.26439/persona2020.n023(1).4833
}

\begin{abstract}
Resumen. Se exploran las representaciones sociales del cuerpo y la salud utilizando redes semánticas y un cuestionario semiestructurado en estudiantes universitarios de nivel socioeconómico bajo. La alimentación, el ejercicio, la higiene y la atención médica son los elementos más importantes de la representación de la salud. Los significados del cuerpo se asocian con estereotipos de género, lo que repercute en el cuidado de la salud. Destaca la importancia de reconocer los atributos, habilidades y manifestación del cuerpo como características humanas, así como el carácter intrínsecamente social del cuerpo y la salud, y no reducirlo al ámbito individual y físico.
\end{abstract}

Palabras clave: cuerpo / salud / género / jóvenes / representación social

\section{MEANINGS OF BODY AND HEALTH IN THE CONTEXT OF MODERN LIFESTYLES}

Abstract. Social representations of body and health were explored among university students of low socioeconomic status using semantic networks and a semi-structured questionnaire. Diet, exercise, hygiene and medical care are the most important elements representing health. The meanings of the body are associated with gender stereotypes, which have an impact on health care. This research highlights the importance of recognizing the attributes, abilities and manifestations of the body as human characteristics, as well as the intrinsically social nature of the body and health, not limiting them to an individual and physical scope

Keywords: body / health / gender / youngster / social representation

Correo electrónico: dania.aguilar@uacm.edu.mx 


\section{INTRODUCCIÓN}

Los estilos de vida actuales implican una cierta idea del cuerpo acorde con los valores y principios de la sociedad moderna. Un cuerpo que también nos alerta frente a los problemas de salud. Es evidente el incremento de enfermedades como la diabetes (segunda causa de mortalidad en México después de las enfermedades cardiovasculares), la obesidad (México ocupa el segundo lugar a nivel mundial) y otras asociadas a los hábitos de alimentación, que se presentan no solo en adultos, sino a edades cada vez más tempranas; así como las problemáticas relacionadas con la salud sexual y reproductiva de los jóvenes.

Tomando en cuenta la relevancia que ha adquirido el cuerpo en el mundo actual, los significados que se le atribuyen nos hablan de las ideas, creencias, emociones, valores y prácticas compartidas socialmente, y que abarcan aspectos tanto individuales (en un sentido físico, mental y emocional) como sociales (en términos del lugar que ocupan las personas y de las diversas actividades que desempeñan en la sociedad), así como el peso que tiene la influencia de un gran número de mensajes y estereotipos que impactan en la manera como las personas conciben y valoran su cuerpo y orientan sus acciones. De manera particular resulta relevante comprender los significados que los jóvenes atribuyen al cuerpo, como fenómeno social característico de las sociedades modernas.

Desde una perspectiva psicosocial, estudiar las representaciones sociales del cuerpo que construyen los jóvenes significa aproximarse a la complejidad de los procesos vinculados a las problemáticas de salud: no solo a los factores inmediatos que subyacen a ellas, sino además a la relevancia de los significados que estos atribuyen al cuerpo y que inciden de manera cotidiana en sus condiciones de salud. Reconocer que el cuerpo está cargado de significados que son construidos socialmente permite conocer los modos de pensar, de actuar y de sentir que corresponden a los estilos de vida en la sociedad actual, que indican la importancia de los procesos socioculturales considerados fundamentales en la construcción psicosocial del sujeto y que descansan en determinadas concepciones de lo humano surgidas en los contextos socioculturales donde se han construido.

\section{El cuerpo como construcción sociocultural en la sociedad moderna}

Como construcción social configurada por el contexto sociocultural, el cuerpo constituye un objeto simbólico y de representación, por medio del cual construimos nuestra relación con el mundo. "Nos referimos al cuerpo como ese espacio complejo en el que se cruzan procesos vitales con figuras del pensamiento, a partir del cual el propio cuerpo humano se ve transformado por las diversas modalidades que adopta" (Muñiz, 2007, p. 67). Al ser constitutivo de nuestra condición humana, el cuerpo no solo alude a las características físicas y procesos fisiológicos propios de nuestra especie, sino que está cargado de 
significados construidos socialmente que nos indican el peso de los procesos de socialización que son fundamentales en la conformación psicosocial del sujeto. Es decir, las personas viven y se vinculan desde sus contextos socioculturales, elaboran sus identidades y construyen su entorno también a partir de sus manifestaciones corporales, en las que el cuerpo es reconocido como fundamental en el ámbito social y cultural.

Podría decirse que el cuerpo contemporáneo ha sido desacralizado por los logros en muchos campos de la medicina y sometido a experimentaciones, pero también el cuerpo es el resultado de la revolución sexual y de los movimientos sociales, surgidos sobre todo en la segunda mitad del siglo pasado, que han cuestionado el papel de la mujer, la moral cristiana y han reivindicado el ejercicio de sus derechos en diversos ámbitos de la sociedad. En contraste, a finales del siglo xx, en el contexto de la crisis de la sociedad moderna, se manifiesta un cambio en los estilos de vida familiares, laborales y educativos, que implica también un cambio en los valores, filosofías de vida y en las formas de relacionarse que adoptan las personas con respecto a sus hábitos de consumo y de entretenimiento, entre otros. Este cambio pone en evidencia la incertidumbre, lo efímero y el vacío que caracteriza a la sociedad moderna en los entornos urbanos y que muestra el hiperindividualismo, el hedonismo, el pragmatismo y la seducción de los objetos de consumo como parte de lo que define la condición humana actual (Lipovetsky, 2002). En concordancia, el cuerpo es también estereotipado en las imágenes a través de la televisión, la publicidad y otros medios de comunicación empeñados en resaltar una perfección obsesiva, e impulsado por una sociedad de consumo en la que participan industrias de diversidad de productos, gimnasios, moda, dietas, deporte, cirugía estética, ejercicio y otras tantas actividades que indican un interés cada vez mayor por el cuerpo. Este interés se ha ido manifestando de manera masiva y actualmente refleja una visión caracterizada por la búsqueda del bienestar personal a través del bienestar del cuerpo (Le Breton, 2010).

En cuestiones de salud, muchos debates éticos se han dado en torno al tratamiento de diversas enfermedades, las terapias que llegan a ser muy agresivas en su afán de curar, la eutanasia, la gestación subrogada, la manipulación genética, incluso respecto a la calidad de la atención médica en los servicios de salud, los cuales reflejan la prevalencia de una concepción del ser humano como "máquina humana" que se resiste a ver al cuerpo de manera integral. Esta concepción es propia de las sociedades occidentales modernas, que adoptaron el modelo cartesiano de relaciones mente-cuerpo, culturanaturaleza, característico del paradigma científico moderno, por lo que se dio "poca atención a la producción social de los cuerpos, así como a la experiencia subjetiva de la relación con el propio cuerpo o el cuerpo del otro" (González, 2008, p. 17). La idea del cuerpo visto como objeto natural lo mantuvo ajeno al sujeto y pasó a un segundo plano convirtiéndose en objeto en sus funciones prácticas, las cuales no se cuestionan por ser consideradas naturales e inherentes a la persona. 
Otro aspecto de la salud en términos sociales reside en el incremento de la tasa de enfermedades como diabetes, padecimientos cardiovasculares y obesidad registrado en México en los últimos años, así como de las enfermedades relacionadas con la salud sexual y reproductiva, que ponen en evidencia los retos a los que nos enfrentamos como sociedad en términos de educación para la salud. Más aún, el contexto actual de la pandemia de la covid-19 nos plantea el reto de formas de convivencia que nos llevan a reflexionar acerca de los estilos de vida modernos y la percepción de riesgos, que sin duda implica nuestra corporalidad e interacciones en la vida cotidiana.

Este estado de cosas coloca en la discusión la necesidad de abordar el cuerpo y la salud no únicamente con relación a la prevención y atención de enfermedades, sino en un sentido más amplio desde las ideas, pensamientos, conocimientos, sentimientos, valoraciones y prácticas, a partir de las cuales las personas les atribuyen una serie de significados, materia de interés de la psicología social.

\section{Género y salud}

En el cuerpo confluyen ideas, valores, roles, sentimientos y expectativas que definen a las personas como seres social y culturalmente construidos desde lo masculino y lo femenino, a partir de la diferencia sexual, lo cual repercute en las prácticas desde las asimetrías de poder que impactan en todos los ámbitos de la vida cotidiana, incluida la salud. En este sentido, se habla de un sistema de sexo/género (Rubin, 1996) que regula y orienta las relaciones entre hombres y mujeres a partir de las diferencias que, en el marco de los contextos socioculturales de nuestra sociedad, atribuyen a las características físicas y funciones fisiológicas una serie de usos y prácticas distintas para hombres y mujeres. Este hecho implica la adjudicación de roles y estereotipos socialmente aceptados que trascienden en todos los ámbitos de la vida de las personas:

Los cuerpos masculino y femenino adquieren diferente categoría, el cuerpo femenino se convierte en una metáfora al representar la naturaleza, la emocionalidad, la irracionalidad y la sensualidad. Las imágenes del cuerpo femenino apetitoso, frágil, guiado por sus emociones, contrastan con el cuerpo masculino concebido como el lugar de la racionalidad y el autocontrol, eje de la idea de superioridad masculina y centro del poder social. (Muñiz, 2007, p. 70)

Las funciones que históricamente se han asignado a las mujeres, vinculadas a la reproducción (engendrar, parir, amamantar), y un papel sociosexual en función del otro han llevado a considerar como natural que ellas deban hacerse cargo del cuidado del otro. Esto rebasa el hecho biológico y ha propiciado la naturalización de las conductas, emociones, prácticas e ideas diferenciadas, vistas como propias de las mujeres, lo cual favorece que se asigne como su ámbito de acción el espacio privado y que su aspecto físico adquiera mayor importancia al tener que ser atractiva y potencialmente reproductiva. En 
cuanto a las funciones atribuidas a los varones, más vinculadas al ámbito público, hacen referencia a las destrezas físicas, la fortaleza corporal, la valentía y el deber de responder en términos de un buen desempeño para el trabajo y otras actividades asimiladas a la masculinidad, hasta convertirse en la base de los estereotipos que acarrean desigualdades entre ambos sexos. Este hecho, además, otorga una mayor valoración y estatus a las actividades consideradas masculinas respecto de las femeninas (Bustos, 2001). Cabe aclarar que las diferencias anatómicas y fisiológicas existentes entre mujeres y hombres no son el origen de las desigualdades, sino las regulaciones sociales que se caracterizan por el ejercicio del control y el poder, que marcan la pauta de dichas desigualdades entre los sexos. Estas impactan en las conductas, los valores, las prácticas y los significados que se asignan al cuerpo y que derivan en estereotipos, limitaciones e inequidades que afectan el pleno desarrollo físico, psicológico y social de las personas.

Los roles de género, entendidos como el conjunto de prescripciones socioculturales sobre lo que es considerado masculino y femenino, no solo orientan las prácticas, sino que constituyen un componente importante en la construcción de las identidades femeninas y masculinas que, a través de los procesos de socialización, lleva a la generación de estereotipos. Estos "se refieren al conjunto de creencias, pensamientos o representaciones acerca de lo que significa ser hombre o mujer, incidiendo en esferas tales como apariencia física, rasgos psicológicos, relaciones sociales, formas de pensar" (Bustos, 2011a, p. 209). En este sentido, se reconoce que un factor determinante de la salud son precisamente los estereotipos de género, tan arraigados en la sociedad, que se producen y reproducen socialmente, y que exaltan aspectos como la belleza física, la fuerza, la energía, entre otros atributos, los cuales contribuyen a hacer del "cuerpo joven" la característica de las sociedades modernas, colocándolo incluso como referente de las etapas posteriores de la vida.

Uno de los aspectos donde se aprecia el impacto de los estereotipos es en la relevancia que ha adquirido el cuerpo en los últimos años, la cual muestra que la experiencia de la interacción social está cada vez más condicionada por la imagen del cuerpo, atravesado por el género, en los modos de pensar, sentir y actuar de las mujeres y los hombres. Los estereotipos inciden en los significados que el cuerpo adquiere y la manera como se construyen las identidades y subjetividades de las personas. En estudios que abordan su repercusión en la manera como se construye la imagen corporal y su relación con problemas de salud, como los trastornos alimentarios, se ha encontrado que las imágenes que se difunden a través de los medios de comunicación no solo son un ideal respecto a la apariencia física, sino que conllevan valores, reconocimientos, emociones y sentimientos vinculados al aspecto físico. De tal manera que si el aspecto físico no coincide con el patrón o estereotipo esperado, se genera insatisfacción respecto de la propia imagen corporal, lo que conduce a la búsqueda de opciones para corresponder con dicho estereotipo (Bustos, 2011a, 2011b; Davis, 2007). 
Los estereotipos de género relacionados con las prácticas de salud también se reflejan, por ejemplo, en el hecho de que en México solamente 3 de cada 10 hombres mayores de 40 años consideren necesario acudir al servicio médico para atenderse o para algún diagnóstico temprano (Cruz, 2014), o los datos que indican que cada año mueren 100000 mujeres en nuestro país por enfermedades cardiovasculares vinculadas a riesgos de salud, como sobrepeso y obesidad, diabetes, anemia, preeclampsia, tabaquismo, depresión y ansiedad, entre otros (Cruz, 2105).

Más aún, se ha señalado que no es solo la salud física la que se identifica a través de un cuerpo cuidado y cultivado, sino incluso la salud mental asume indicadores estéticos (González, 2008). Por ejemplo, en un estudio realizado por Bustos (2011b), se encontró que el $68 \%$ de las mujeres que siguen dietas restrictivas no lo hacen para cuidar su salud, sino por su imagen corporal, ya que las personas delgadas, sobre todo las mujeres, se perciben como guapas, exitosas, felices, con prestigio y poder. Este estado de cosas nos alerta sobre la necesidad de abordar tales problemáticas no únicamente por sus consecuencias en la salud, sino desde una perspectiva más amplia, ya que lo que las personas piensan, conocen, sienten, valoran y hacen indica las representaciones sociales que construyen respecto al cuerpo y la salud.

\section{El cuerpo y la salud desde las representaciones sociales}

La teoría de las representaciones sociales propuesta por Serge Moscovici en 1961 estudia los modos en que las personas explican y dan sentido a la realidad cotidiana como formas de conocimiento social compartido y construido desde sus contextos socioculturales. Las representaciones sociales contribuyen a la comprensión de los significados que las personas construyen respecto del cuerpo y la salud, los cuales forman parte de su vida cotidiana y orientan muchas de sus decisiones. Es decir, las representaciones sociales se ocupan del estudio de los modos de conocimiento y de los procesos simbólicos en relación con las acciones y manifestaciones concretas (Wagner y Hayes, 2011), que en este caso se refieren al cuerpo y la salud.

El concepto de representación social concierne a la manera como aprehendemos los acontecimientos de la vida diaria, las características de nuestro ambiente, las informaciones que en él circulan, las personas de nuestro entorno próximo o lejano; esto es, se refiere al conocimiento del sentido común (Moscovici y Hewstone, 1986). Este conocimiento se construye a partir de nuestras experiencias, pero también de las informaciones, conocimientos y modelos de pensamiento que aprendemos y transmitimos a través de la tradición, la educación y las diversas formas de comunicación. Por tanto, se trata de un conocimiento socialmente elaborado y compartido con el que las personas intentan dominar su entorno, comprender y explicar los hechos e ideas de su universo de vida, actuar sobre y con otras personas, situarse respecto de ellas, responder a las 
preguntas que le plantea el mundo, etcétera. Al participar en la construcción social de nuestra realidad, estamos ante un conocimiento práctico que da sentido, dentro de un incesante movimiento social, a acontecimientos y actos que terminan por sernos habituales y que forjan las evidencias de nuestra realidad.

En este sentido, el cuerpo se ha estudiado desde los procesos sociales y los contextos específicos que van configurando las distintas maneras de construirlo, mirarlo y relacionarse. Para Jodelet (1984), el sentimiento personal que se tiene hacia el cuerpo es lo que lo hace un punto de referencia para las personas en sus vidas, ya que son las normas sociales y las demandas culturales lo que regula la experiencia que se tiene con el cuerpo, como es el caso del deseo y el dolor, dos ámbitos profundamente personales. En este sentido, el cuerpo:

Es el objeto de la experiencia inmediata, el lugar donde se inscribe la subjetividad, donde los afectos y las inversiones psicológicas se proyectan. Por otra parte, es el objeto de un tipo de pensamiento social regulado por prescripciones acerca de cómo mantener y presentarse [...] en la escena social. Por lo tanto, el cuerpo constituye un área privilegiada para estudiar la interacción de los procesos individuales y colectivos en la elaboración de las representaciones sociales. (Jodelet, Ohana, Bessis-Monino y Dannenmüller, 1982, citado por Wagner y Hayes, 2011, p. 106)

En las discusiones actuales sobre los significados de la corporalidad, se reconoce que los usos, prácticas y discursos sobre el cuerpo comunican tanto sensaciones físicas como significados socioculturales. Es decir, lo que las personas hacen o no desde lo corporal puede comunicar las especificidades de cada cultura y, simultáneamente, lo que es común a la condición humana. Si bien el cuerpo puede abordarse como un espacio individual y material, no puede aislarse sin tomar en cuenta la diversidad de características y contextos sociales. Por ello debe tenerse en cuenta que las percepciones, experiencias y construcciones del cuerpo nos hablan de este incluso como un sitio particular de poder y control social y político, como lo señaló Michel Foucault (2009, 1993, 1979) a lo largo de su obra.

El cuerpo colocado como lugar emblemático de la individualidad es, además, el receptor de las normatividades y regulaciones socioculturales de género, clase, edad, etnia, dejando entrever una multiplicidad de dimensiones y significados. Como señala Jodelet (1984), el cuerpo aparece, por un lado, como mediador social del puesto de la persona en la sociedad y, por otro, como mediador del conocimiento de sí mismo y de otros; es decir, la representación que se forma la persona de sí misma en el aspecto físico, mental y emocional está relacionada con la percepción del propio cuerpo. Asimismo, considera que estudiar el cuerpo desde la perspectiva de las representaciones sociales contribuye al estudio de las formas colectivas de ver y experimentar el cuerpo y los comportamientos relacionados, por lo que plantea que dicha teoría puede contribuir a la comprensión del cuerpo más allá de la dimensión psicológica individual, 
aportando conocimientos con la recuperación del cuerpo como construcción social en diversos campos, como el de la salud.

El cuerpo y la salud cobran importancia porque son parte de la vida cotidiana de las personas. Nos hablan de las condiciones sociales en las que estas viven, de sus formas de pensar y reproducir los significados que tienen, lo que los hace un objeto psicosocial. En este sentido, "la teoría de las representaciones sociales intenta establecer precisamente ese vínculo entre la sociedad y la mentalidad individual" (Wagner y Hayes, 2011, p. 15). Así, las prácticas que se van arraigando en la vida cotidiana ponen en evidencia un modelo que opera no solo como referente de las condiciones de salud, sino como significados y representaciones que se articulan en la subjetividad y la identidad de las personas. Los cuerpos que no se ajustan a tales expectativas llegan a ser considerados como indicadores de crisis profundas en la estructura social, es decir, el cuerpo como síntoma de desviación, no solo en el sentido de la falta o descuido en la salud, sino como reflejo de los valores éticos y estéticos socialmente aceptados (González, 2008).

Dado que las representaciones sociales no solo inciden en la manera de entender la realidad, sino también en su construcción efectiva, desempeñan un papel importante en la comunicación social y en la producción de significados, así como en la conformación de las identidades personales y sociales, y en la expresión y configuración de los grupos. En otras palabras, estar con personas que ven el mundo como lo vemos nosotros no solo permite establecer relaciones más relajadas, cercanas y satisfactorias, sino que proporciona mayor confianza respecto a la validez de los criterios propios y de la bondad de nuestra forma de ser. En cuanto a las relaciones intergrupales o de sectores de la sociedad, como es el caso de las mujeres y los hombres, la imagen que se forman los grupos respecto de los otros orienta las relaciones que se establecen entre ambos. Como señala lbáñez (1988), la identidad de un grupo o sector social no puede definirse si no es por su diferencia con otros grupos; tanto las personas como los grupos se ubican en el campo social construyendo identidades que son gratificantes, compatibles con los sistemas de normas y valores sociales. En este sentido, las representaciones sociales desempeñan "un papel importante en el control social ejercido por la colectividad sobre cada uno de sus miembros, en particular en los procesos de socialización" (Abric, 2001, p. 16), como ocurre en las representaciones sociales de lo femenino y lo masculino, la salud y la enfermedad, en grupos específicos de población.

Finalmente, las representaciones sociales también ayudan a conseguir que las personas acepten la realidad social instituida, haciendo que se integren de manera satisfactoria en la condición social que supuestamente les corresponde en función de criterios sociales como los roles y estereotipos de género. Al igual que las ideologías, aunque de forma mucho más concreta, las representaciones sociales contribuyen tanto a la legitimación y fundación del orden social como al cambio y la transformación. En este sentido, 
la perspectiva de género "como un sistema ideológico cuyos distintos procesos orientan el modelaje de la representación social diferenciada de los sexos, determinando formas específicas de conducta asignadas en función del sexo biológico" (Flores, 2001, p. 7), contribuye a explicar las diferencias socialmente elaboradas y asignadas a las mujeres y hombres como un proceso de construcción social, por lo que se reconoce su importancia en el estudio de las representaciones sociales.

Se plantea que este sistema, "en tanto que normativiza la diferencia de roles entre los sexos, no puede existir separado de las prácticas sociales que ambos sexos reproducen" (Flores, 2001, p. 6). Sin embargo, "no se trata de negar la existencia de diferencias entre los sexos, sino de cuestionar su origen y construcción cultural" (Flores, 2001, p. 19). En este caso, indagar las representaciones sociales del cuerpo requiere revisar la construcción de género en la formación de los estereotipos vinculados a él.

El estado de la salud que se registra en las últimas décadas en México demanda no solo investigaciones dentro del ámbito médico, sino también estudios que contribuyan a identificar las maneras en que se conciben el cuerpo y la salud en la sociedad actual. Es decir, implica colocar a la persona antes que a la enfermedad y aproximarse a los significados y representaciones sociales que se construyen a este respecto. En este sentido, además de considerar a todos los sectores de población, de manera específica es necesario atender a aquellos en los que la prevención y el aprendizaje de hábitos saludables a temprana edad resultan significativos para lograr mejores condiciones de salud a lo largo de su vida, como es el caso de los jóvenes. Es ampliamente conocido que muchas enfermedades crónicas de la edad adulta, como diabetes, hipertensión o afecciones cardiacas, tienen su antecedente en etapas tempranas porque se relacionan con hábitos y prácticas que las favorecen. Incluso en años recientes se ha observado el desarrollo de padecimientos crónico-degenerativos a edades cada vez más prematuras (INSP, 2012; Imjuventud, 2010).

A partir de lo expuesto, resulta necesario conocer los significados que se atribuyen al cuerpo y la salud desde los contextos en que se desenvuelven los jóvenes, en este caso, aquellos que se ubican en entornos urbanos de pobreza, falta de servicios y espacios para su pleno desarrollo físico y psicoemocional. Para ello se realizó un estudio cualitativo cuyo objetivo fue conocer las representaciones sociales de jóvenes universitarios de ambos sexos y escasos recursos acerca del cuerpo y la salud, e identificar las características e implicaciones de dichas representaciones sociales desde una perspectiva de género. Este estudio forma parte de una investigación más extensa (Aguilar, 2015), por lo que se reporta solo lo referido al cuerpo y la salud. 


\section{MÉTODO}

\section{Participantes}

Los participantes fueron en total 102 jóvenes ( 55 mujeres y 47 hombres), con edades de 18 a 29 años, estudiantes de licenciatura de la Universidad Autónoma de la Ciudad de México (UACM), del plantel San Lorenzo Tezonco, el cual atiende a casi la mitad de estudiantes de dicha universidad. La UACM es una institución pública de educación superior cuyo perfil de estudiantes, en su mayoría, se ubican en los niveles de media y alta marginación socioeconómica. La mayoría de los participantes (87 \%) viven en colonias y barrios con niveles de marginalidad alta y muy alta ${ }^{1}$ de la Ciudad de México, además de un $6 \%$ que proviene de varios municipios conurbados ${ }^{2}$ con niveles también de marginalidad alta y muy alta. El promedio de edad fue de 21,8 años en las mujeres y de 23,6 años en los hombres.

\section{Instrumentos}

Se usó la técnica de asociaciones libres que incluyó la utilización de dos instrumentos. Uno, referido a redes semánticas naturales (Valdez, 1998), que consistió en una lista que exploró cinco conceptos: mi cuerpo, salud, cuerpo sano, cuerpo enfermo y autocuidado; y un cuestionario semiestructurado que abordó aspectos relativos a las prácticas de autocuidado que realizan los jóvenes en su vida cotidiana, la percepción del propio cuerpo, así como frases incompletas referidas a los gustos, sentimientos, valoraciones y los aspectos que consideran más relevantes respecto al cuerpo y la salud.

\section{Procedimiento}

La aplicación se realizó en el salón de clase de los grupos participantes, conformados por estudiantes de ambos sexos, a quienes previamente se les solicitó su participación voluntaria y se les dieron las instrucciones correspondientes. Posteriormente, se proporcionó a cada estudiante los instrumentos mencionados con la indicación de no abrirlos hasta que se les diera el aviso. Primero, se les pidió llenar la hoja de datos generales y luego responder uno por uno los conceptos estímulo del formulario de redes semánticas, según las instrucciones explicadas. Una vez concluida esta parte, se les dijo que respondieran el cuestionario semiestructurado. Al término de la aplicación se recogieron los instrumentos y se agradeció su participación.

1 De acuerdo con el índice de marginación de la Ciudad de México del Sistema de Información del Desarrollo Social (SIDESO), Secretaría de Desarrollo Social del GDF (www.sideso.df.gob.mx/index. php?id=35).

2 Municipios: Nezahualcóyotl, Los Reyes La Paz, Ixtapaluca y Valle de Chalco Solidaridad. 


\section{RESULTADOS}

Se encontró una gran riqueza semántica en los cinco conceptos revisados, esto es, el total de palabras definidoras para cada concepto, la cual resulta mayor entre las mujeres en gran parte de los conceptos, como se muestra en la tabla 1.

Tabla 1

Riqueza semántica de los conceptos

\begin{tabular}{lcc}
\hline \multirow{2}{*}{ Concepto } & \multicolumn{2}{c}{ Riqueza semántica } \\
\cline { 2 - 3 } & Mujeres & Hombres \\
\hline Mi cuerpo & 230 & 206 \\
Salud & 207 & 223 \\
Cuerpo sano & 235 & 195 \\
Cuerpo enfermo & 248 & 210 \\
Autocuidado & 267 & 219 \\
\hline
\end{tabular}

En términos generales, los significados atribuidos al cuerpo son valorados de manera positiva. Se reconocen en principio algunas partes del cuerpo como ojos, manos, cabeza, salud, corazón, así como atributos físicos que indican una estrecha relación entre las representaciones sociales del cuerpo y la salud, en las cuales se identifica la relevancia de los estereotipos de género, ya que la apariencia física y los atributos se orientan a las características estéticas, sobre todo entre las mujeres, y de desempeño entre los hombres. Para ellas las palabras relacionadas con el cuerpo son belleza, manos, piernas, gordo, movimiento, cabello y piel, que culturalmente están relacionadas con estereotipos femeninos que destacan aspectos como delgadez, arreglo personal, tener una piel lisa, tersa, firme y sin estrías, así como rasgos emocionales y de conducta definidos por palabras como delicada, tierna, orientada a los afectos; además, se consideran responsables de su cuidado y de los otros, es decir, ser un cuerpo para los demás.

Los varones, en cambio, se refieren al cuerpo como máquina o instrumento. Se mencionan palabras como dedos, fuerza, huesos, manos, ejercicio, brazos, lo cual se orienta en un sentido utilitario y de rendimiento para el desempeño de sus actividades, lo que evidencia también el peso de los estereotipos de género. Asimismo, se encontró que el $31 \%$ de las mujeres desearían estar más delgadas, mientras que el $21,3 \%$ de los varones esperarían estar más robustos o musculosos, lo que apunta a la relevancia de la expectativa social respecto al cuerpo vinculada a los estereotipos de género y que propicia el deseo de alcanzar el ideal estético considerado como deseable para cada sexo. Este aspecto nos remite a que la experiencia de la interacción en la sociedad actual está cada vez más condicionada por la imagen del cuerpo y la expectativa en los modos 
deseables de sentir y actuar atribuidos a las mujeres y a los hombres, los cuales inciden en la subjetividad y las identidades que se construyen. Esto coincide con lo que señala Vigarello (2005) respecto al lugar del cuerpo joven, principalmente en los contextos urbanos, que marca la pauta de lo que se considerará un cuerpo sano o enfermo. Dichas representaciones sociales están orientadas por la valoración del vigor físico, el tamaño, la robustez, la energía, el poder muscular, frente a la fragilidad, la debilidad y pequeñez, considerados obstáculos para la potencia necesaria requerida para el trabajo.

Si bien en ambos grupos se reconoce que en el cuerpo se manifiestan malestares físicos y emocionales, para los hombres la relación con este aspecto es problemática, ya que la visión utilitaria del cuerpo en el imaginario masculino propicia una distancia emocional que limita la manifestación de las experiencias corporales respecto de los afectos y las emociones, los cuales son considerados más próximos a las mujeres y la feminidad. En otros casos, para muchos hombres permanece un sentimiento impreciso o vago a la hora de identificar la experiencia de su cuerpo (Cornell, 2003), e incluso pueden llegar a negar todo referente corporal frente al sufrimiento y los conflictos o padecimientos cotidianos.

La gran mayoría de participantes de ambos sexos se consideran sanos (95\%); $\sin$ embargo, destaca la referencia que se hace de la obesidad, a la que se reconoce como un problema relacionado más con la estética, sobre todo entre las mujeres, que como un problema de salud. Cabe señalar que no es solo la salud física la que se identifica a través de un cuerpo cuidado y cultivado, sino incluso la salud mental asume indicadores estéticos (González, 2008). Así, Bustos (2011b) señala que el 68 \% de las mujeres que siguen dietas restrictivas no lo hacen para cuidar su salud, sino para mejorar su imagen corporal, ya que las personas delgadas, sobre todo las mujeres, se perciben como guapas, exitosas, felices, con prestigio y poder. En consecuencia, se produce cada vez más una búsqueda del bienestar personal a través del bienestar del cuerpo, ya que este, al ser reconocido de manera más directa por su condición física, concreta y objetiva, es considerado el lugar de manifestación de la salud, así como de malestares físicos y emocionales.

En ambos grupos, el significado de la salud se asocia con aspectos como la alimentación, el ejercicio, la higiene (más valorada por las mujeres), la prevención y los cuidados (que incluyen la atención médica), pasando por actividades vinculadas al placer, el descanso y lo lúdico. También, a características y atributos físicos relacionados con elementos estético-sexuales (belleza, placer, satisfacción, sexo) y de índole utilitaria o productiva (fuerza, energía, resistencia), así como los psicoemocionales (felicidad, bienestar, amor, cariño) y actividades intelectuales (estudiar, leer, aprender).

La alimentación se identifica como el factor más importante para la salud en ambos grupos. Las mujeres manifiestan mayor riqueza semántica, incluso distinguen entre lo 
que consideran una alimentación sana, buena o balanceada de lo que definen como mala alimentación, mencionando cierto tipo de alimentos como frutas, verduras, cereal, vitaminas y beber agua. En cambio, los hombres, si bien señalan algunos alimentos como carnes, carbohidratos, frutas y verduras, se refieren a la alimentación en general y hacen poca diferencia entre lo que consideran como buena o mala alimentación. La centralidad de la alimentación en la representación social de la salud coincide con estudios que indican que la alimentación saludable es frecuente entre personas con estudios universitarios (Andreatta, 2013), aunque en los jóvenes la alimentación también está asociada con el placer y el hecho social de la comida (Aguirre, 2000). Esto contrasta con estudios que reportan que las prácticas de consumo y las representaciones de los alimentos entre las clases sociales más bajas están orientadas hacia productos rendidores, así como la elección está en función de la actividad que se desempeñe. Entonces, entre los sectores de menos recursos económicos, los alimentos, además de ser rendidores, deben ser baratos, saciar y aportar energía, es decir que son de alto contenido calórico, contrario a los criterios de elección en sectores económicos altos, que buscan alimentos considerados sanos, ligeros y sabrosos (Aguirre, 2000; Bourdieu, 2012). En este caso, aunque se trata de jóvenes de escasos recursos, se diferencian en que son estudiantes universitarios y, por tanto, tienen mayor acceso a informaciones respecto a la alimentación y la salud, lo que les permite reconocer cierto tipo de productos vinculados a la mala alimentación.

En ambos grupos se reportaron más palabras asociadas al cuerpo enfermo que al cuerpo sano. El cuerpo enfermo tiene una connotación negativa, sobre todo entre los varones, y destaca la importancia de las expectativas sociales de género sobre lo que se entiende por ser hombre y desempeñar un rol masculino. El cuerpo enfermo se relaciona principalmente con la mala alimentación, que se define como chatarra, comida rápida, grasa, refrescos o bebidas con mucha azúcar, siendo las mujeres las que hacen mayor mención a la mala alimentación. Entre los hombres, la salud implica, además, no fallar, ya que estar enfermos significa no poder responder conforme a la expectativa socialmente esperada para ellos; es decir, el cuerpo enfermo preocupa no por la enfermedad en sí, sino por las consecuencias negativas que les genera en términos del incumplimiento de sus obligaciones y responsabilidades laborales. De ahí que palabras como chatarra, basura, inservible, inutilidad, señaladas por los varones, estén relacionadas con el cuerpo enfermo en su condición funcional o instrumental, lo que repercute, a su vez, en su valoración y percepción personal.

Asimismo, la expresión de emociones y sentimientos es más evidente en la representación social del cuerpo enfermo en ambos grupos. En el caso de la enfermedad, se identifica una mayor asociación de palabras que expresan emociones y sentimientos como tristeza, depresión, angustia, enojo, dolor, malestar, desesperación, etcétera. Más aún, para los hombres, la enfermedad se relaciona con hospital, cama y muerte. Esto hace referencia a la poca atención a la prevención y a la manifestación temprana de síntomas; 
por el contrario, la gravedad de estos es lo que alerta para la atención médica. También se mencionan los accidentes, el consumo de tabaco, alcohol y otras sustancias adictivas como causas de enfermedad o incluso de muerte en esta etapa. Estos significados indican que hace falta poner atención y desarrollar estrategias para el reconocimiento tanto de síntomas como de sentimientos y emociones asociados al cuerpo y la salud, principalmente entre los varones, que favorezca el autocuidado y se incorpore como práctica ética del cuidado de sí.

En el caso de las mujeres, estar enfermas se asocia con dependencia, desorden, indisciplina, irresponsabilidad, aunque también se reconoce como parte de la condición de vida. Tales palabras se relacionan con la demanda hacia las mujeres como responsables de su salud. Además, reconocen en mayor medida otros riesgos para la salud como los embarazos no planeados y las infecciones de transmisión sexual.

Tanto las mujeres como los hombres consideran importante cuidar el cuerpo y la salud. Sin embargo, los motivos para su cuidado están más orientados a la presencia estética en el caso de las mujeres y al desempeño en las actividades en el caso de los hombres. Esto evidencia el peso de los estereotipos de género en el significado que se atribuye al cuerpo y la salud. Asimismo, para las mujeres la atención médica es una práctica común vinculada a la salud y el cuerpo sano, no así en el caso de los hombres, para quienes la atención médica está más relacionada con estar enfermo, por lo que acudir al médico solo resulta necesario si se tiene un problema de salud que lo amerite.

Un aspecto destacable en ambos grupos es que el cuerpo y la salud son considerados un asunto personal; por tanto, su cuidado es visto como una tarea y responsabilidad individual. No obstante la importancia del entorno social y la participación colectiva, como elementos clave para el cuidado del cuerpo y la salud se reconoce principalmente a la familia y el apoyo que ella significa, siendo las mujeres las que se sienten más comprometidas a atender a algún familiar enfermo, pero también a cuidarse a sí mismas para no enfermar y no molestar a su familia. En menor grado se identifican palabras como derecho, seguro y gratuita, que colocan a la salud como un asunto de compromiso y responsabilidad del Estado.

También se hace referencia al entorno social de riesgo, por ejemplo, las mujeres mencionan violencia, vigilancia y respeto; mientras que los hombres hablan de inseguridad y accidentes. Pero también en ambos grupos se reconoce la importancia de la educación en temas de salud con palabras como conocimiento, conciencia, obligación, reflexión e investigar. De igual manera, se mencionan palabras como amor, conciencia y responsabilidad, en el caso de los hombres; y descanso y fuerza, en el caso de las mujeres. Ellas, aunque con una menor mención, indican diferencias con respecto a la expectativa asociada con los estereotipos masculinos y femeninos tradicionales en la valoración del cuerpo y la salud. 


\section{CONCLUSIONES}

Como fenómeno característico de las sociedades modernas, aproximarse al estudio de las representaciones sociales del cuerpo y su relación con la salud permite identificar la diversidad de significados que se les atribuyen y asumir una postura crítica respecto de los estereotipos de género. En estos, el cuerpo, convertido en objeto, es el centro de atención en una cultura visual como la que vivimos, inserta en una sociedad moderna caracterizada por el individualismo que impulsa la idea de que el individuo es el actor principal y responsable único de atender sus propias necesidades de salud.

Es necesario fortalecer el carácter intrínsecamente social del cuerpo y la salud para contrarrestar las ideas que lo reducen al ámbito individual, físico y de responsabilidad personal, así como reconocer que los cambios en la sociedad implican también cambios en los modos de vivir y experimentar lo social a través del cuerpo. Impulsar prácticas más efectivas de cuidado del cuerpo y la salud, junto con el desarrollo de estrategias que no se limiten a las posibilidades individuales, sino que busquen una participación más activa del entorno familiar, escolar y de otras instancias vinculadas con la salud. Esto revela la necesidad de atender la trascendencia del entorno social y revisar el papel que juegan las instituciones educativas y de salud para la atención y prevención de diversas problemáticas, como los hábitos de alimentación que pueden provocar enfermedades crónico-degenerativas que se presentan cada vez a edades más tempranas, así como otros problemas relacionados con la salud sexual y reproductiva en los jóvenes.

En este sentido, las estrategias educativas deben incluir la discusión y reflexión acerca de los significados que se atribuyen al cuerpo y la salud no solo entre los jóvenes, sino en los diversos sectores de la sociedad, incluidos los profesionales de la salud, ya que, en el caso de la práctica médica, por ejemplo, se involucran las propias representaciones que se tienen respecto a sí mismo, a los jóvenes, el cuerpo y la salud. Estas representaciones influyen en la atención y las recomendaciones que se dan están asociadas a tales conceptos. Por tanto, se debe buscar promover prácticas y formas de interacción que favorezcan una mejor relación con el propio cuerpo y un mejor cuidado de la salud.

Finalmente, para mejorar el desarrollo no solo de los jóvenes, sino de todas las personas, es indispensable conocer y recuperar la riqueza y variedad de significados que se construyen en torno al cuerpo, así como sus posibilidades de manifestación y reconocimiento como características humanas. Es decir, reconocer que los atributos y estereotipos asignados a las mujeres y los hombres han tenido impactos en la salud, al ser considerados como factores de riesgo, lleva a plantear que, en la medida que se generen cambios en tales estereotipos y se reconozca que las maneras de pensar, de actuar, las emociones, sentimientos y demás atributos son características humanas, ello repercutirá en la construcción de representaciones sociales que favorezcan mejores prácticas para el cuidado de la salud y el desarrollo integral de las personas. 


\section{REFERENCIAS}

Abric, J. C. (2001). Prácticas sociales y representaciones. México D. F.: Ediciones Coyoacán.

Aguilar, D. (2015). Las representaciones sociales del cuerpo y su relación con la salud y el autocuidado (tesis de maestría). Universidad Nacional Autónoma de México, Facultad de Psicología.

Aguirre, P. (2000). Los alimentos rendidores y el cuerpo de los pobres. En A. Millán (Comp.), Arbitrario cultural. Racionalidad e irracionalidad del comportamiento comensal (pp. 799-820). Huesca: La Val de Onsera.

Andreatta, M. M. (2013). La alimentación y sus vínculos con la salud desde la teoría de las representaciones sociales. Revista Diaeta, 31(142), 42-49.

Bourdieu, P. (2012). La distinción. Criterio y bases sociales del gusto. México D. F.: Taurus.

Bustos, 0. (2001). Género y socialización: familia, escuela y medios de comunicación. En M. A. González y J. Mendoza (Comps.), Significados colectivos: procesos y reflexiones teóricas (pp. 289-357). México D. F.: Instituto Tecnológico y de Estudios Superiores de Monterrey, campus Estado de México; CIIACSO.

Bustos, O. (2011a). Estereotipos sexistas e imagen corporal en la publicidad comercial y gubernamental. En R. Trejo y A. Vega (Coords.), Diversidad y calidad para los medios de comunicación (pp. 205-219). México D. F.: Asociación Mexicana de Derecho a la Información.

Bustos, O. (2011b). Los medios y la construcción de género: factor de riesgo para trastornos alimentarios como anorexia y bulimia. Derecho a Comunicar, 2, 60-79.

Cornell, R. (2003). Masculinidades. México D. F.: Programa Universitario de Estudios de Género, UNAM.

Cruz, A. (12 de diciembre del 2014). Solo 3 de cada 10 hombres acuden al médico oportunamente para diagnóstico de males. La Jornada, p. 50.

Cruz, A. (21 de septiembre del 2015). Males cardiovasculares causan la muerte de 100000 mujeres al año. La Jornada, p. 37.

Davis, K. (2007). El cuerpo a la carta. Estudios culturales sobre cirugía cosmética. México D. F.: La Cifra.

Flores, F. (2001). Psicología social y género. El sexo como objeto de representación social. México D. F.: McGraw-Hill; UNAM.

Foucault, M. (2009). Vigilar y castigar. Nacimiento de la prisión. México D. F.: Siglo xxI Editores.

Foucault, M. (1993). Historia de la sexualidad I. México D. F.: Siglo Xxı Editores.

Foucault, M. (1979). Microfísica del poder. Madrid: Ediciones de la Piqueta. 
González, C. (2008). Identidad y percepción social del cuerpo. En T. Porzecanski (Comp.), El cuerpo y sus espejos (pp. 17-31). Montevideo: Planeta.

Ibáñez, T. (1988). Ideologías de la vida cotidiana. Barcelona: Sendai.

Instituto Mexicano de la Juventud (Imjuventud). (2010). Encuesta Nacional de la Juventud. México D. F.: Autor. Recuperado de http://www.imjuventud.gob.mx

Instituto Nacional de Salud Pública (INSP). (2012). Encuesta Nacional de Salud y Nutrición, ENSANUT 2012. México D. F.: Autor. Recuperado de http://ensanut.insp.mx

Jodelet, D. (1984). The representation of the body and its transformations. En R. Farr $y$ S. Moscovici (Eds.), Social representations (pp. 211-238). Cambridge: Cambridge University Press.

Le Breton, D. (2010). Antropología del cuerpo y modernidad. Buenos Aires: Nueva Visión.

Lipovetsky, G. (2002). La era del vacío. Barcelona: Anagrama.

Moscovici, S., y Hewstone, M. (1986). De la ciencia al sentido común. En S. Moscovici (Comp.), Psicología social II (pp. 679-710). Buenos Aires: Paidós.

Muñiz, E. (2007). Cuerpo y corporalidad: lecturas sobre el cuerpo. En M. Á. Aguilar (Coord.), Tratado de psicología social (pp. 67-95). México D. F.: Anthropos; UAM Iztapalapa.

Rubin, G. (1996). El tráfico de mujeres: notas sobre la economía política del sexo. En M. Lamas (Comp.), El género: la construcción cultural de la diferencia sexual (pp. 35-96). México D. F.: Porrúa; Programa Universitario de Estudios de Género, UNAM.

Valdez, J. L. (1998). Las redes semánticas naturales, usos y aplicaciones en psicología social. México D. F.: Universidad Autónoma del Estado de México.

Vigarello, G. (2005). Corregir el cuerpo. Historia de un poder pedagógico. Buenos Aires: Nueva Visión.

Wagner, W., y Hayes, N. (2011). El discurso de lo cotidiano y el sentido común. La teoría de las representaciones sociales. Barcelona: Anthropos. 
